\title{
O GÊNERO (IN)VISÍVEL DA TERCEIRA IDADE NO SABER DA ENFERMAGEM
}

Maria do Livramento Fortes Figueiredo* Maria Antonieta Rubio Tyrrell**

\section{Resumo}

Trata-se de um estudo bibliográfico, no qual realizou-se um levantamento da produção científica de enfermagem referente à Mulher na Terceira Idade no Catálogo de Dissertações e Teses do CD-room do Centro de Estudos e Pesquisas em Enfermagem(CEPEn) da Associação Brasileira de Enfermagem - ABEn no período de 1979 a 1999. Objetivou conhecer os afastamentos e/ou aproximações no saber de enfermagem com o objeto de estudo da tese de doutorado: os saberes e práticas de saúde das mulheres idosas, na perspectiva de gênero. Concluiu-se que a mulher na terceira idade foi investigada em poucos estudos, além do mais, os objetos destas investigações se afastaram fortemente das questões de gênero, demonstrando-se o aspecto assexuado da velhice, e negação dos papéis sociais da mulher. Descritores: mulher idosa; gênero; saber de Enfermagem

\begin{abstract}
It is a bibliographical study in which the scientific production of Nursing regarding Women in Old Age was surveyed. The study was based on the Catalog of Dissertations and Theses from the CD-ROM of the Center for Studies and Research on Nursing (CEPEn) of the Brazilian Nursing Association (ABEn), from 1979 to 1999. It was aimed at getting to know the deviations and/or approximations in the knowledge of Nursing by way of the object of study of the Ph.D. thesis 'Health practice and knowledge of old women in the gender perspective'. It was found out that old women have been investigated in few studies. In addition, the objects of these investigations have strongly deviated from gender issues, demonstrating the sexless aspect of old age and the denial of women's social roles.

Descriptors: old women; gender; knowledge of Nursing

Title: The (in)visible gender of old age in the knowledge of Nursing
\end{abstract}

\section{Resumen}

Se trata de un estudio bibliográfico en el cual se realizó un levantamiento de la producción científica de enfermería referente a la Mujer en la Tercera Edad, en el Catálogo de Disertaciones y Tesis del CD - room del Centro de Estudios e Investigaciones en Enfermería (CEPEn) de la Asociación Brasileña de Enfermería - ABEn en el período de 1979 a 1999. El objetivo es conocer los alejamientos $y$ aproximaciones en el saber de enfermería con el objeto de estudio de la tesis de doctorado: los saberes y prácticas de salud de las mujeres ancianas, en la perspectiva de género. Se concluye que la mujer en la tercera edad, fue objeto de estudio en pocas investigaciones $y$ aquellas investigadas se alejaron mucho de las cuestiones de género, demostrándose el aspecto asexuado de la vejez y negación de los papeles sociales de la mujer.

Descriptores: mujer vieja; género; saber de Enfermería

Título: El Género (in) visible de la Tercera Edad en el Saber de Enfermería

\section{Introdução}

Estando inscrita no Curso de Doutorado da Escola de Enfermagem Anna Nery - EEAN da Universidade Federal do Rio de Janeiro - UFRJ, desenvolvendo a tese: A mulher e o tempo - saberes e práticas de saúde na terceira idade, na perspectiva de gênero, e buscando contextualizar a problemática e identificar convergências e aproximações, bem como, divergências e descontinuidades na produção de enfermagem referente à temática desse estudo, além de procurar aproximações dos referenciais teóricos e metodológicos que estão sendo utilizados no desenvolvimento desta tese, buscou-se levantar a produção científica da enfermagem contida no CD-ROM do Centro de Estudos e Pesquisas em Enfermagem(CEPEn) ${ }^{(1)}$ da Associação Brasileira de Enfermagem - ABEn no período de 1979 a 1999.

Constatou-se que produção científica de enfermagem referente à terceira idade ainda é pequena, e é menor ainda quando se trata de estudos com enfoque nas questões de gênero, desta forma se evidencia uma negação das relações de gênero. As funções e papéis característicos da condição feminina nos estudos pesquisados apresentam-se de forma velada ou (in)visível na terceira idade.

Partindo desta constatação, além de ter feito um levantamento minucioso da produção de enfermagem referente à terceira idade, busquei construir este artigo, a partir de reflexões sobre a mulher na terceira idade, enfocando tanto nos aspectos demográficos, sociais e culturais, como aqueles referentes às condições de saúde e doença a que estão submetidas às mulheres idosas e assim contribuir com uma produção do conhecimento que venha melhorar a assistência de enfermagem a este grupo populacional que mais cresce no Brasil e no Mundo, procurando evidenciar a relevância das questões de gênero na determinação das condições de saúde e na qualidade de vida da mulher na terceira idade.

\section{Metodologia}

Trata-se de um estudo bibliográfico, no qual realizou-se um levantamento da produção científica de enfermagem referente à Mulher na Terceira Idade no Catálogo de Dissertações e Teses do CD-ROM do Centro de Estudos e Pesquisas em Enfermagem(CEPEn) ${ }^{(1)}$ da Associação Brasileira de Enfermagem - ABEn no período de 1979 a 1999.

A busca foi procedida utilizado-se os seguintes unitermos: idoso, terceira idade, mulher idosa, velhos, velhas, mulher, gênero. Foram identificados trabalhos nas categorias: idoso e mulher idosa. Foram levantados 54 resumos de dissertações e teses na área temática da terceira idade.

\section{Apresentação e discussão dos resultados}

A produção científica em enfermagem no Brasil é recente, tendo seu início com as exigências da carreira universitária em 1963 e intensificando-se, a partir dos anos setenta, com a regulamentação da pós-graduação stricto sensu, e mais recente ainda quando se trata em investigar um fenômeno tão novo como é o envelhecimento populacional, principalmente, quando se focalizar como objeto de investigação da enfermagem outra categoria de análise considerada extremamente contemporânea para as ciências sociais, como são as questões de gênero( ${ }^{(2)}$.

No Brasil, como em toda parte, a mulher é objeto de

\footnotetext{
* Enfermeira. Professora Assistente da Universidade Federal do Piauí. Mestre em Enfermagem pela EEAN/UFRJ e Doutoranda da Escola de Enfermagem Anna Nery / UFRJ. Este artigo foi produzido na Disciplina Pensamento Contemporâneo II do Curso de Doutorado em Enfermagem da EEAN/UFRJ, ministrada pelas Professoras e Doutoras: Ivis Emília de Oliveira Souza e Marléa Chagas Moreira. **Enfermeira. Professora Doutora, Titular da EEAN/UFRJ. Diretora da EEAN/UFRJ. Orientadora da Tese de Doutorado.

E-mail do autor: livramento.figueiredo@bol.com.br
} 
preconceitos, cristalizados em papéis, mais ou menos estereotipados. A questão da mulher como tema de pesquisas e estudos na área acadêmica ganhou particular relevo na década de 70 , quando aumentou consideravelmente o número de programas criados em universidades e centros de investigação, nos EUA e em numerosos países da Europa ${ }^{(3)}$.

Nesse contexto alguns temas atraíram o interesse de numerosos estudiosos das ciências sociais, tais como, o trabalho feminino, a sexualidade, a violência, e já no final do século a participação política da mulher, principalmente com a Constituição de 1988, e posteriormente, com as leis eleitorais, estabeleceu-se o regime de cotas para as candidaturas de mulheres para os parlamentos, porém os temas Gênero e Terceira Idade, ainda não obtiveram toda atenção que merece, principalmente na área da saúde, em especial na Enfermagem.

Porém com a mudança no perfil demográfico mundial e brasileiro, cresce o interesse pelos estudos sobre a velhice, principalmente na medicina social, que trouxe como conseqüência a investigação na área social, focalizando a velhice-problema, a velhice falta de dinheiro, da solidão, da aposentadoria e da viuvez, constituindo-se assim como objeto desses estudos; no entanto, esses investigações limitavamse a enfocar a velhice como um período da vida em que ocorre uma diminuição das áreas de relacionamento social e de perdas físicas, emocionais e sociais.

O interesse pelo estudo da velhice, não da velhiceproblema, da velhice escondida, asilada, doente, mas da velhice nas suas relações sociais, nas suas experiências de vida, nas suas possibilidades e na sua cidadania, vem surgir de fato na década de 70, no âmbito da atual sociedade urbana brasileira(4).

Portanto, como já era de se esperar ser pequena a produção científica em Enfermagem abordando, Gênero e Envelhecimento, apesar de serem considerados dois fenômenos sociais de grande repercussão na saúde pública, mas que também passaram a ser foco de atenção das ciências somente a partir da década de 60.

Das 54 produções científicas levantadas no CD-ROM do CEPEn-ABEn ${ }^{(1)}(1979$ - 1999) referente à temática da terceira idade, 48 (quarenta e oito) das Dissertações de Mestrado e Teses de Doutorado foram produzidas a partir de 1988, o que demonstra ser relativamente, recente a preocupação de enfermeiros e pesquisadores, com a problemática do envelhecimento. Estas 54 produções se distribuem da seguinte forma: 45 são dissertações de mestrado, sendo 37 têm como sujeito o idoso, e 08 a mulher idosa. Já as Teses são 09, sendo 08 destas estudando o idoso independente de sexo, e apenas 01 investiga a mulher idosa. Dos estudos que tem como sujeitos de investigação a mulher, em apenas 02 (dois) foram enfocadas as questões de gênero.

Também se evidenciou uma concentração desses estudos nas academias de três estados brasileiros, São Paulo (27 trabalhos), Santa Catarina (08 trabalhos) e Rio de Janeiro (07 trabalhos), o que vem confirmar o desenvolvimento da produção científica de enfermagem nestes centros de qualificação profissional stricto sensu, que foram pioneiros nas modalidades: mestrado e doutorado.

É por este motivo que, o artigo referente ao levantamento da produção científica de Enfermagem na área da terceira idade é intitulado: O gênero in(visível) da terceira idade no saber da Enfermagem pois até aqueles estudos que têm como sujeitos à "mulher" enfocaram com maior ênfase os aspectos biológicos, fisiológicos e patológicos do envelhecimento feminino, não considerando os aspectos sociais e culturais estabelecidos pela sociedade para mulher em qualquer faixa etária.

Sobre a importância de se pesquisar o envelhecimento, na perspectiva de gênero e evidenciar as relações de gênero com o envelhecimento, ressalta-se os achados de Miller ${ }^{(5: 13)}$ em estudo sobre a saúde das mulheres idosas na América Latina e Antilhas, quando o autor salienta que dois fatores intervêm intensamente na qualidade de vida das idosas: o primeiro é as relações de poder e os papéis femininos de subordinação; o segundo é a participação das idosas em grupos cujas atividades sejam capazes de incentivá-las a utilizar suas potencialidades.

Ao analisar os resumos selecionados na temática da terceira idade, considerando a proposta de categorização temática e de linhas de pesquisa(6) da produção científica em Enfermagem, em que pese à escassez de informações dos resumos, para melhor elucidação categorial dos estudos analisados, foi possível chegar a seguinte distribuição por categoria:

1. Na categoria assistencial foram identificados 35 (trinta e cinco) resumos que corresponderam $64,9 \%$ dos estudos de enfermagem sobre a terceira idade, estes apresentaram como foco de estudo os aspectos relacionados ao cuidado ou assistência de Enfermagem ${ }^{(7)}$.

2. Na categoria organizacional foram identificados 14 (quatorze) resumos representando $25,9 \%$ dos estudos selecionados, mostrando que estas duas dimensões do trabalho de Enfermagem, assistencial e a organizacional ou gerencial, são vistas pelos enfermeiros de serviços como indissolúveis(8).

3. A categoria profissional referente atuação da Enfermeira, como profissional, pautada em um referencial teórico e metodológico para uma sistematização própria na assistência. Nesta categoria evidenciou-se o menor número de estudos, apenas 05 (cinco) correspondendo a 9,20\% dos estudos pesquisados, o que mostra sermos de fato uma profissão, que enquanto ciência está em construção.

Analisando as abordagens utilizadas nestas produções, identifica-se que as mesmas apresentam-se marcadas pelos referenciais positivistas predominantes nas pesquisas em saúde neste contexto temporal em que foram realizadas. Também se percebe uma forte valorização da pesquisa quantitativa, abordando principalmente os aspectos orgânicos do ser humano numa perspectiva funcionalista. Das 54 (cinqüenta e quatro) produções cientificas 35 (trinta e cinco), ou seja, $64,8 \%$ apresentaram o positivismo como predominante nas suas fundamentações teóricas, apenas 19 (dezenove) destes estudos mostram-se com análises mais qualitativas pautadas nas ciências sociais.

Dois desses trabalhos que apresentaram uma abordagem das questões de gênero, ambos são produções da Escola de Enfermagem Anna Nery - EEAN/UFRJ, dos anos de 1998 e 1999, isto também evidencia as inovações, os novos paradigmas e métodos implantados nos Programas de PósGraduação da EEAN/UFRJ, que passam a enfocar referenciais das ciências humanas e sociais, tais como, o marxismo e a fenomenologia.

Os enfoques sociais e culturais, característicos dos referenciais das ciências humanas e sociais, não eram, até então, considerados como relevantes nas investigações em saúde e mais especificamente, na área de enfermagem, principalmente, em relação a uma temática relativamente nova para sociedade brasileira, como a da terceira idade, ainda mais nesta perspectiva de gênero, que busco desvendar na minha tese de doutorado em andamento.

Para melhor visualização será apresentado quadro sintético ilustrando o levantamento feito no CD-ROM do CEPEn/ $\mathrm{ABEn}^{(1)}(1979$ - 1999).

O recorte temporal no qual foi feito o levantamento destas produções aponta para um contexto de situações paradoxais, nas quais os técnicos e pesquisadores, ainda não conseguiram desvendar as problemáticas sanitárias ligadas à infância e adolescência, e já têm como desafio preocuparem-se com o crescimento demográfico da terceira idade, e com as problemáticas e contextos deste grupo populacional. 


\section{Reflexões sobre o binômio: Gênero x Terceira Idade}

Para falar do gênero (in)visível na terceira idade, devese antes de tudo, conceituar gênero, que não significa apenas as diferenças biológicas entre os sexos masculino e feminino, mas sobretudo, representa os valores e os papéis sociais e culturais que os homens e mulheres têm na sociedade. O gênero não significa o mesmo que sexo, ou seja, enquanto o sexo se refere à identidade biológica de uma pessoa, gênero está ligado à sua construção social como sujeito masculino ou feminino(9)

O gênero é mais do que uma identidade aprendida, sendo constituído e instituído pelas múltiplas instâncias e relações sociais, pelas instituições, símbolos, formas de organização social, discursos e doutrinas. Pensando assim, devemos entender que essas diversas instâncias sociais são instituídas pelos gêneros e também os instituem; elas são generificadas ${ }^{(9)}$

Neste sentido vale ressaltar que a identidade de gênero parece ser realmente, constitutiva da identidade (geracional) de idosas. As trajetórias sociais de gênero vêm demonstrando ser determinantes, na situação real e nos sentimentos dessas pessoas idosas, ultrapassando, não raro, a diversidade de situação de classe, quando homens e mulheres vêem-se colocados, diferencialmente, quanto às possibilidades e os sentimentos de bem-estar, liberdade e auto-realização na velhice $^{(10-11)}$.

No processo do envelhecimento feminino, os dados mostram que, em 1980, havia em escala mundial, três homens de 65 anos e mais para cada quatro mulheres, relação que se mostra ainda mais forte nos países desenvolvidos, em razão do grande número de homens mortos durante a Segunda Guerra Mundial. Na verdade, quanto mais a idade aumenta, mais as mulheres são numerosas; o envelhecimento passa a ser um fenômeno que se conjuga, antes de tudo, no feminino ${ }^{(12)}$.

Diferentes expectativas sociais nortearam, então, a trajetória desses homens e mulheres de mais idade. E com tal intensidade, que os diferenciais de gênero obscurecem ou ultrapassam, com freqüência, as diferenças de classe desses velhos e velhas de hoje. Também é por isso que, a categoria gênero assume uma grande importância nos estudos e análises da Terceira Idade, visto ser uma possibilidade de clarificação porque este grupo social é limitado a grupo etário por autoridades governamentais, profissionais de saúde e pela própria sociedade.

Nesse dinâmico processo de mudança do ciclo vital na estrutura demográfica, econômica, política, enfim, na maneira de viver e de estabelecer relações sociais. Pelo menos três mudanças são especialmente relevantes para lançar luz sobre os novos papéis sociais do/a velho/a na atualidade e suas novas formas de sociabilidade: mudanças na estrutura etária da população, na sócio-econômica e nos papéis dos sexos/ gêneros ${ }^{(13)}$

No tocante as condições de saúde a mulher também é atingida de forma diferenciada do homem, e em muitas destas doenças se evidenciam as questões de gênero como fundamentais em sua determinação, a exemplo disto, a depressão de maior ocorrência entre as mulheres, na grande maioria das vezes desencadeada a partir de conflitos das relações sociais entre homens e mulheres.

A análise conclusiva feita por Fajardo ${ }^{(14)}$ retrata a realidade das mulheres na terceira idade caracterizada pela vulnerabilidade física e econômica na sociedade. Essa caracterização deve-se a três fatores: primeiro, por sua maior morbidade, resultante de diferenças fisiológicas que se vêem agravadas pelo efeito acumulado da desnutrição, as gestações contínuas, o desgaste físico e psicológico de uma dupla jornada (doméstica e trabalhista), e sua subordinação social e econômica. Segundo, porque esta vulnerabilidade tem aumentado pela solidão que acompanha a maior longevidade com respeito ao seu parceiro. $E$ terceiro, por seu menor acesso às prestações de serviço de seguridade social e de saúde, devido a sua posição desvantajosa no mercado de trabalho durante a idade ativa, isto é, as menores taxas de emprego, particularmente no setor formal da economia, sua participação trabalhista intermitente por causa da maternidade, e sua menor remuneração.

Esses dados, na perspectiva Latino - Americana, se retratam também no Brasil. Para agravar o quadro de saúde da mulher na terceira idade, no país, a situação apresenta-se contraditória: os Programas Nacionais de Saúde da Mulher, como o Programa de Assistência Integral à Saúde da Mulher $\operatorname{PAISM}^{(15)}$, não contemplam ações assistenciais dirigidas à mulher idosa e os recentes Programas de Assistência aos Idosos não prestam assistência específica à Mulher; desconhecendo assim, a questão de gênero na terceira idade.

\begin{tabular}{|c|c|c|c|c|c|c|c|c|}
\hline Enos Estados & SP & RS & SC & BA & RJ & PB & MG & Total \\
\hline 1979 & & 1 & & & & & & 1 \\
\hline 1980 & & & 1 & & & & & 1 \\
\hline 1981 & & 1 & & & & & & 1 \\
\hline 1982 & 1 & & & & & & & 1 \\
\hline 1985 & & & & & 2 & & & 2 \\
\hline 1988 & & & & 2 & & & & 2 \\
\hline 1989 & 1 & & & & & 1 & & 2 \\
\hline 1990 & 3 & & $2^{\mathrm{b}}$ & & & & & 5 \\
\hline 1993 & $4^{\mathrm{a}}$ & & 1 & 2 & & & & 7 \\
\hline 1995 & 2 & & & 1 & 2 & & & 5 \\
\hline 1996 & 2 & & & & & & & 2 \\
\hline 1997 & $3^{\mathrm{a}}$ & & 1 & & & 2 & & 6 \\
\hline 1998 & 1 & & $3^{\mathrm{b}}$ & & $1^{\mathrm{c}}$ & & 1 & 6 \\
\hline 1999 & 10 & & & & $2^{\mathrm{c}}$ & $1^{\mathrm{d}}$ & & 13 \\
\hline $\begin{array}{c}\text { TOTAL PI } \\
\text { ESTADO }\end{array}$ & 27 & 2 & 8 & 5 & 7 & 4 & 1 & 54 \\
\hline
\end{tabular}

Quadro 1 - Distribuição da Produção em Enfermagem por ano de realização e por estado brasileiro.

\section{Considerações Finais}

Após a discussão dos resultados do levantamento da produção científica de enfermagem referente ao idoso no CD room do CEPEn/ABEn ${ }^{(1)}$ no período de 1979 a 1999, evidenciouse que a mulher na terceira idade aparece como sujeito de poucos estudos, além do mais, os enfoques e os objetos destas investigações se afastam fortemente das questões de gênero, demonstrando-se o aspecto assexuado da velhice, além da negação dos papéis sociais impostos à mulher, independente da idade, classe social, etnia e religião.

Articulando estas análises sobre a produção intelectual em enfermagem, referente à terceira idade, com as reflexões feitas

a São Paulo - SP (1993) - Duas destas produções sobre a mulher idosa, (1997) e (199) - Uma produção sobre a mulher idosa em cada ano.

B Santa Catarina - SC nos anos 1990 e 1998 apresenta uma produção enfocando a mulher idosa.

c Rio de Janeiro - RJ - no ano de 1998 e 1999, duas destas produções tratam da mulher idosa e enfocam as questões nas análises dos seus resultados.

d Paraíba - PB - no ano de 1999, uma Dissertação de Mestrado teve a Mulher Idosa como sujeito do estudo. 
por estudos contemporâneos de feministas e pesquisadoras das ciências sociais e antropológicas, percebe-se a necessidade e a demanda de problemas a serem investigados pela enfermagem com a utilização de referenciais inovadores capazes enfocar o ser humano de maneira integral, tendo também como base suas vertentes sociais, culturais e psicológicas.

Desta forma se reverte de grande importância e relevância, a produção de um conhecimento que venham contribuir para reorientar a prática de ensino e assistencial na área de enfermagem a população idosa, principalmente a feminina, é o grupo populacional que mais cresce no Brasil e no Mundo e também o que apresenta a maior demanda de problemas sociais e de saúde, exigindo portanto, uma formação de recursos humanos para saúde, principalmente, na enfermagem capaz de desenvolver a atenção integral à saúde.

E assim possibilitar aproximações e articulações com aspectos importantes decorrentes das mudanças nos papéis dos sexos/gêneros ocorridos, nas últimas décadas ${ }^{(13)}$. Tais mudanças têm alterado o modo de vida e o comportamento das pessoas também de mais idade, sobretudo das mulheres idosas, à medida que são elas as principais responsáveis pela reconstrução da velhice em nossa sociedade, abrindo espaço para novos processos de sociabilidade, ficando evidente a necessidade de profissionais e pesquisadores visualizarem as questões de gênero em seus estudos por serem determinantes das condições de cidadania e da qualidade de vida na terceira idade.

\section{Referências}

1. Associação Brasileira de Enfermagem. Centro de Estudos e Pesquisas em Enfermagem. Catálogo de Dissertações e Teses do Centro de Estudos e Pesquisas em Enfermagem(CEPEn) no período de 1979 a 1999. Brasília: ABEn:2000 [trabalhos em CD-ROM].

2. Rocha SMM, Silva GB. Linhas Filosóficas e Ideológicas na Pesquisa em Enfermagem no Brasil. Revista Brasileira de Enfermagem, Brasília (DF) 1987 out/dez;40(4):217.

3. Tabak F. Autoritarismo e participação política da mulher. Rio de Janeiro: Edições Graal; 1983.

4. Barros MML. Testemunho de vida: um estudo antropológico de mulheres na velhice. In: Barros MML, organizadora. Velhice ou Terceira Idade? Rio de Janeiro: Fundação Getulio Vargas; 1998.
5. Miller LS. Envelhecer na América Latina. A saúde do mundo. Genebra: OMS;1990.

6. Carvalho V. Linhas de Pesquisa e Prioridades de Enfermagem Proposta de distinção gnoseológiga para o agrupamento da produção científica da Pós-Graduação em Enfermagem. Documento apresentado no Fórum Nacional de Coordenadores de Programas de Pós-Graduação em Enfermagem, realizado durante XVI Enf Nordeste e III Encontro de Pesquisa da RENE Salvador (BA); 2000. $11 \mathrm{f}$. (Digitado).

7. Moriya TM. A pesquisa no ensino de Pós-Graduação em Enfermagem. In: Pós-Graduação Strictu Sensu em Enfermagem: um estudo do seu desenvolvimento no Brasil. Ribeirão Preto (SP): Fundação Instituto de Ribeirão Preto;1998.p.61-76.

8. Lima MAD, Gustavo AS, Coelho DF, Schmitz UV, Rosa RB. Concepç̃es de enfermeiras sobre seu trabalho no modelo clínico de atenção à saúde. Revista Brasileira de Enfermagem, Brasília (DF) jul/ago 2000, 53(3):343-54.

9. Louro GL. Nas redes do conceito de gênero. In: Lopes MJM, Meyer DE, Waldow VR. Gênero \& Saúde. Porto Alegre (RS): Artes Médicas; 1996.p.9-13.

10. Motta AB. Falando em surdina: são mulheres velhas. Belo Horizonte (MG): ABEP;1994.p.231-144.

11. Debert GG. Gênero e Envelhecimento. Estudos Feministas, Rio de Janeiro 1994;2(3):62.

12. Peixoto $C$. De volta às aulas ou De como ser estudante aos 60 anos. In: Veras R, organizador. Terceira Idade: Desafios para o Terceiro Milênio. Rio de Janeiro: Relume Dumará;1997.p.55.

13. Gomes MQC. Velhice: a busca de novos espaços de sociabilidade. In: Amaral CCG, Sales CMV, Azevedo HS, D'Ávila SMG, orgnizadoras. Múltiplas Trajetórias: Estudos de Gênero. Fortaleza (CE);REDOR/ NEGIF UFC;2001.p.209.

14. Fajardo ML. A Situação de Saúde da Mulher na América Latina e Caribe - uma análise com a ótica de gênero. Goiânia (GO): OPAS/ OMS;1997.p.20-34.

15. Ministério da Saúde (BR). Programa de Assistência Integral à Saúde da Mulher - PAISM. Brasília (DF): Centro de Documentação; 1984.

Data de recebimento: $12 / 08 / 04$

Data de Aprovação: 22/12/2004 